\title{
Heat and Mass Transfer for Hartmann and Dufour' s Effects on Irreversibilities at Double-Diffusive Natural Convection in a Square Cavity
}

Mounir Bouabid ${ }^{1 *}$, Nejib Hidouri' ${ }^{1}$ Mourad Magherbi ${ }^{2}$ and Ammar Ben Brahim ${ }^{1}$

${ }^{1}$ Chemical and Process Engineering Department, Engineers National School of Gabès, Gabès University, Tunisia

${ }^{2}$ Chemical Engineering Department, High Institute of Applied Sciences and Technology, Gabès University, Tunisia

\begin{abstract}
In this paper, entropy generation of double-diffusive natural convection in a 2D dimensional enclosure with magnetic and Dufour effects has been numerically performed. Dirichlet boundary conditions for temperature and solute concentration are applied to the two vertical walls of the enclosure; wheras the two horizontal walls are adiabatic and insulated. The governing equations of continuity, momentum, energy and concentration are numerically solved by using a Control Volume Fined Elements Method, CVFEM of Patankar. The governing parameters of the problem are the thermal Grashof number $\left(G_{T}\right)$, the buoyancy ratio $(N)$, the Hartmann number $(\mathrm{Ha})$, the Dufour parameter $(D u)$ and the Prandtl number $(P r)$. The obtained results were presented graphically via the velocity field components, temperature and concentration distributions, entropy genertion rate behaviour and by isotherms, streamlines and isentropic lines maps. The average Nusselt and Sherwood numbers are also derived and discussed numerically. The investigated results showed that the flow field and then entropy generation are notably influenced by the considering parameters.
\end{abstract}

Keywords: MHD double-diffusive convection; Square cavity; Entropy generation; Dufour effect; CVFEM

\section{Nomenclature}

$B$

C

c

$\mathrm{C}_{\mathrm{p}}$

$D$

$D_{F}$

$\mathrm{Du}$

E

F

g

$\mathrm{Gr}_{\mathrm{T}}$

$\mathrm{Gr}$

$\mathrm{Ha}$

J

$\mathrm{J}_{\mathrm{k}}$

$\mathrm{L}$

Le

$\mathrm{N}$

$\mathrm{Nu}$

$\mathrm{P}$

$\mathrm{P}$

$\operatorname{Pr}$

$\mathrm{Ra}_{\mathrm{T}}$

$\mathrm{Sc}$

Sh

$S$

$\mathrm{T}$

$t, \tau$

$\mathrm{u}, \mathrm{v}$
Magnetic Field (T)

Dimensionless Concentration

Concentration $\left(\mathrm{mol} \cdot \mathrm{m}^{-3}\right)$

Specific Heat $\left(\mathrm{J} \cdot \mathrm{Kg} \cdot \mathrm{K}^{-1}\right)$

Molecular Diffusivity $\left(\mathrm{m}^{2} \cdot \mathrm{s}^{-1}\right)$

Dufour coefficient $\left(\mathrm{m}^{2} \cdot \mathrm{s}^{-1} \cdot \mathrm{K}^{-1}\right)$

Dufour parameter

Electric Field $\left(\mathrm{V} \cdot \mathrm{m}^{-1}\right)$

Electromagnetic Force $(\mathrm{N})$

Gravitational Acceleration $\left(\mathrm{m} \cdot \mathrm{s}^{-2}\right)$

Thermal Grashof Number

Solutal Grashof Number

Hartmann Number

Electric Current Density $\left(\mathrm{A} \cdot \mathrm{m}^{-2}\right)$

Diffusion Flux $(k=u, v, T)$

Length of the Cavity (m)

Lewis number

Buoyancy Ratio

Nusselt Number

Dimensionless Pressure

Pressure $\left(\mathrm{N} \cdot \mathrm{m}^{-2}\right)$

Prandtl Number

Thermal Rayleigh Number, RaT $=$ GrT.Pr

Schmidt Number

Sherwood Number

Entropy Generation Rate $\left(\mathrm{J} \cdot \mathrm{s}^{-1} \cdot \mathrm{K}^{-1} \cdot \mathrm{m}^{-3}\right)$

Dimensionless Temperature

Time (s)

Velocity Components in X And Y Directions $\left(\mathrm{m} \bullet \mathrm{s}^{-1}\right)$

$\mathrm{U}, \mathrm{V}$
$\mathrm{U}_{\mathrm{d}}$
$\alpha_{\mathrm{T}}$
$\varnothing$
$\beta_{\mathrm{T}}$
$\beta_{\mathrm{c}}$
$\lambda$
$\lambda_{\mathrm{i}}$
$\mu$
$\mu_{\mathrm{sp}}$
$\mathrm{W}$

$\mathrm{x}, \mathrm{y}$
$\mathrm{X}, \mathrm{Y}$
Greek letters
$\nu$
$\rho$
$\sigma$
$\sigma$
$\theta$

Dimensionless Velocity Components Dimensionless Velocity Vector Thermal Diffusivity $\left(\mathrm{m}^{2} \bullet \mathrm{s}^{-1}\right)$ Magnetic Inclination Angle $\left(^{\circ}\right)$ Thermal Expansion Coefficient $\left(\mathrm{K}^{-1}\right)$ Solutal Expansion Coefficient $\left(\mathrm{m}^{3} \cdot \mathrm{mol}^{-1}\right)$ Thermal Conductivity $\left(\mathrm{J} \cdot \mathrm{m}^{-1} \cdot \mathrm{s}^{-1} \cdot \mathrm{K}^{-1}\right)$ Irreversibility Coefficient $(\mathrm{i}=1,2,3,4)$

Dynamic Viscosity $\left(\mathrm{kg} \cdot \mathrm{m}^{-1} \cdot \mathrm{s}^{-1}\right)$

Chemical Potential Of Species $\left(\mathrm{J}_{\bullet} \mathrm{kg}^{-1}\right)$

Thermodynamic Parameter

$\mathrm{W}=\left[\left(\mathrm{C}_{0} \cdot \theta_{0} / \mathrm{CP}\right] .\left(\partial \mu_{\mathrm{sp}} / \partial \mathrm{C}\right) \theta_{0}, \mathrm{C}_{0}\right.$

Cartesian Coordinates (m)

Dimensionless Cartesian Coordinates

$$
\begin{aligned}
& \text { Kinematic Viscosity }\left(\mathrm{m}^{2} \cdot \mathrm{s}^{-1}\right) \\
& \text { Mass Density }\left(\mathrm{kg} \cdot \mathrm{m}^{-3}\right) \\
& \text { Dimensionless Entropy Generation } \\
& \text { Electric Conductivity }\left(\Omega^{-1} \cdot \mathrm{m}^{-1}\right) \\
& \text { Temperature }(\mathrm{K})
\end{aligned}
$$

*Corresponding authors: Mounir Bouabid, Chemical and Process Engineering Department, Engineers National School of Gabès, Gabès University, Tunisia, Tel: +21625286647; E-mail: bouabid.mpcshun@yahoo.fr

Received October 09, 2017; Accepted October 30, 2017; Published November 01,2017

Citation: Bouabid M, Hidouri N, Magherbi M, Brahim AB (2017) Heat and Mass Transfer for Hartmann and Dufour's Effects on Irreversibilities at Double-Diffusive Natural Convection in a Square Cavity. J Adv Chem Eng 7: 180. doi: 10.4172/20904568.1000180

Copyright: (c) 2017 Bouabid M, et al. This is an open-access article distributed under the terms of the Creative Commons Attribution License, which permits unrestricted use, distribution, and reproduction in any medium, provided the original author and source are credited. 
$\theta_{0}$

\section{Subscripts}

$a$

Mag

$c$

$h$

\section{Introduction}

In the past few years, the problems of double-diffusive convective and heat transfer flows through a cavity under the influence of a magnetic field have been attracted the attention of a number of researchers because of their several applications in many engineering problems, such as in thermal energy storage systems, industrial magnetic devices, petroleum industry, polymer technology and glass fiber production. The interest for analysing the double-diffusive convective flows induced by the cross thermodiffusion effects has surged in view of its importance in many engineering problems such as in insulation, the underground disposal of nuclear wastes and drying processes.

Trevisan and Bejan [1] reported a fundamental numerically and analytically study of scale analysis relative to heat and mass transfer within a vertical slot filled with a porous medium submitted to horizontal combined and pure temperature and concentration gradients. The more observed effect was due to heat transfer and then to mass transfer. They obtained that the magnetic effect is more pronounced than friction one. The magnetic field parameter suppresses the flow in the cavity and this lead to a decrease of entropy generation. At local level, results show that entropy generation lines are localized on lower heated and upper cooled regions of the active walls.

Other related numerical heat and mass transfer studies of a steady free convection in MHD micropolar electrically conducting fluid flow on a vertical plate in the presence of Soret and Dufour effects under the influence of an applied uniform magnetic field were considered by Ali and Alam [2], they observed that the magnetic field exerts a retarding force on the free convection flow, the temperature profile increases as Prandtl and Dufour parameter increases and the concentration increases for increasing values of Shmidt and Soret parameter. Subhakar et al. [3] considered the effect of linear thermal stratification in stable stationary ambient fluid on steady MHD convective flow of a viscous incompressible electrically conducting fluid along a moving, non-isothermal vertical plate in the presence of mass transfer, Soret and Dufour effects and heat generation or absorption, they have found that the local skin-friction coefficient, local heat and mass transfer rates at the plate increase with an increase in the buoyancy forces or Soret or Dufour numbers or heat generation/absorption parameter. The local skin-friction coefficient and the local Nusselt number decreases with increasing the Dufour number (decreasing Soret number) while the local Sherwood number increases. Sharma et al. [4] analyzed The Soret and Dufour effects on unsteady MHD mixed convection flow past an infinite radiative vertical porous plate embedded in a porous medium in the presence of chemical reaction, they have obtained that An increase in radiation parameter, the velocity and temperature profiles increase, with increasing heat source parameter, the velocity and temperature profiles are increasing. The velocity and concentration profiles decrease as an increase in chemical reaction parameter. For fluids with medium molecular weight $\left(\mathrm{H}_{2}\right.$, air), Dufour and Soret effects should not be neglected. Bouabid et al. [5] have studied the contributions of thermal, diffusive, friction and magnetic terms on entropy generation are investigated. Hidouri et al. [6] have reported the influence of Soret and Dufour effects on entropy generation dealing with transient doublediffusive convection of a binary gas mixture for the special case of opposing buoyancy forces with equal intensity. It was found that for moderate thermal Grashof number, Soret and Dufour parameters induced a slight increase of entropy generation, but for relatively higher thermal Grashof number, oscillatory behavior of entropy generation was obtained.

Magherbi et al. [7] have determined numerically the entropy generation due to heat transfer, mass transfer and fluid friction in steady state for laminar double-diffusive convection, in an inclined enclosure with heat and mass diffusive walls.

The more observed effect was due to heat transfer and then to mass transfer. They obtained that the magnetic effect is more pronounced than friction one. The magnetic field parameter suppresses the flow in the cavity and this lead to a decrease of entropy generation. At local level, results show that entropy generation lines are localized on lower heated and upper cooled regions of the active walls.

Recently, Malashetty and Gaikwad [8] have analyzed the effect of cross diffusion namely Soret coefficient and Dufour coefficient on the double diffusive convection in an unbounded vertically stratified two component system with compensating horizontal thermal and solute gradients. They obtained the effect of the Soret coefficient, $S$ is more significant compared to the Dufour effect. The fluid particle moves with greater velocity and high heat transfer rate for the high value of Dufour coefficient and the velocity is less and high mass transfer rate for the presence of Soret coefficient when the left active concentration wall C and right wall $\mathrm{C}_{\mathrm{h}}$. The opposite behavior was observed for the inversed configuration.

Ahammad and Mollah [9] have dealt numerically with MHD free convection flow and mass transfer over a stretching sheet including the Dufour and Soret effects with a magnetic field, the have concluded that for the combined effects of Dufour inreasing and Soret decreasing, the temperature profiles increase while velocity and concentration profiles decrease. The local skin-friction coefficient and the local Nusselt number decreases with increasing the Dufour number (decreasing Soret number) while the local Sherwood number increases. Despite all these studies, the analysis of thermodynamics second law with cross thermodiffusion Dufour and Soret effects at MHD mixed convection, for an electrically conducting fluid have received few attentions. Hence, the aim of this study is to investigate the entropy generation on steady magneto double-diffusive convection in a square confined cavity filled with an incompressible Newtonian binary gas mixture fluid under Dufour's effect in the presence of magnetic field. It is assumed that the enclosure is heated and concentrated at their vertical walls in the presence of a transverse magnetic field.

\section{Mathematical formulation}

Double-diffusive natural convective flow inside a vertical square cavity of length, L, filled with a binary perfect gas mixture composed of air and a pollutant species in the presence of an oriented magnetic field is considered as shown in Figure 1. Different temperature and concentrations are imposed between the left $\left(\theta_{h}, c_{h}\right)$ and right vertical walls $\left(\theta_{c}, c_{c}\right)$, where the $\theta_{h}>\theta_{c}$ and $c_{h}>c_{c}$. Adiabatic and impermeable boundary conditions are imposed on the remaining walls. The gravity acts in the downward direction. It is also assumed that the enclosure is permeated by a uniform magnetic field, $B=B_{x} e_{x}+B_{y} e_{y}$ of constant magnitude $B_{0}$, where $B_{x}$ and $B_{y}$ are space-independent, ex and ey are unit vectors in Cartesian coordinate system. $U=u_{x}+v_{y}$ is the field velocity. The orientation of the magnetic field forms an angle $\emptyset$ with 


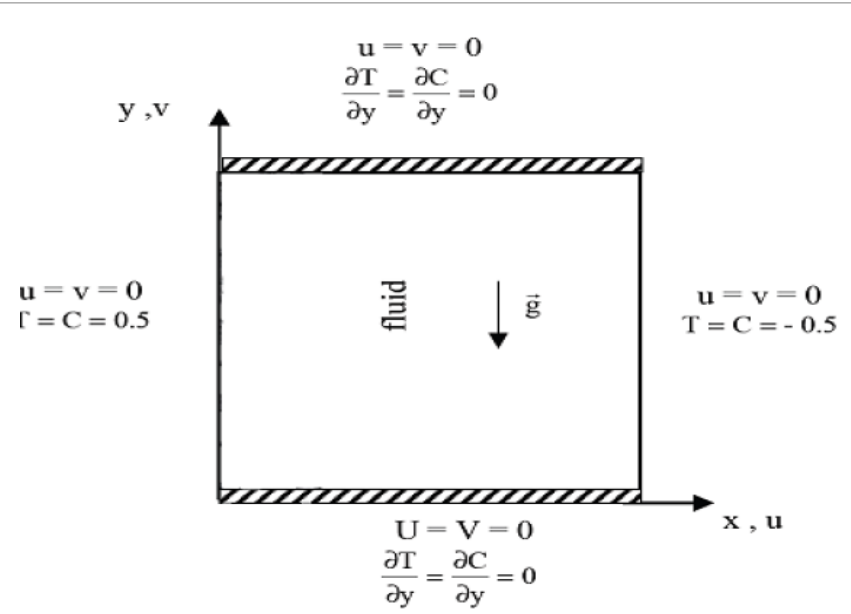

Figure 1: Schematic view of the physical model and coordinate system.

horizontal axis. The electric current, $\mathrm{J}$, and the electromagnetic force, $\mathrm{F}$, are defined by $\mathrm{J}=\sigma_{\mathrm{e}}(\mathrm{V} \times \mathrm{B})$ and $\mathrm{F}=\sigma_{\mathrm{e}}(\mathrm{V} \times \mathrm{B}) \times \mathrm{B}$, respectively. The solution of the governing conservation equations of mass, momentum, energy and the chemical species describing the phenomenon is obtained by considering the following assumptions:

The flow in the cavity is laminar and two-dimensional.

The Soret effect is neglected.

The binary fluid is assumed to be Newtonian, incompressible and to satisfy the Boussinesq approximation.

All physical properties of the fluid are assumed to be constant, except its density variations upon temperature and concentration which is described by the following state equation:

$$
\rho(\theta, c)=\rho_{0}\left[1-\beta_{\mathrm{T}}\left(\theta-\theta_{0}\right)-\beta_{\mathrm{c}}\left(\mathrm{c}-\mathrm{c}_{0}\right)\right] \rightarrow(1)
$$

$\rho_{0}, \theta_{0}$, and $c_{0}$ are the mass density, the temperature and the concentration at reference conditions, respectively. and are the thermal and the solute expansion coefficients, respectively.

$$
\begin{aligned}
& \beta_{C}=-\frac{1}{\rho_{o}}\left(\frac{\partial \rho}{\partial C}\right)_{P} \rightarrow(2.2) \\
& \beta_{C}=-\frac{1}{\rho_{o}}\left(\frac{\partial \rho}{\partial C}\right)_{P} \rightarrow(2.2)
\end{aligned}
$$

Under these above assumptions, the governing equations for the continuity, momentum, and energy and species diffusion in laminar incompressible flow can be written as follows:

$\frac{\partial u}{\partial x}+\frac{\partial v}{\partial y}=0 \rightarrow(3)$

$\frac{\partial u}{\partial t}+u \frac{\partial u}{\partial x}+v \frac{\partial v}{\partial y}=-\frac{1}{\rho_{0}} \frac{\partial p}{\partial x}+v \nabla^{2} u+\frac{\sigma_{e} B_{0}^{2}}{\rho_{0}}(v \cos \phi-u \sin \phi) \sin \phi \rightarrow$ (4)

$\frac{\partial v}{\partial t}+u \frac{\partial v}{\partial x}+v \frac{\partial v}{\partial y}=-\frac{1}{\rho_{0}} \frac{\partial p}{\partial y}+v \nabla^{2} v-\frac{\rho}{\rho_{0}} g+\frac{\sigma_{e} B_{0}^{2}}{\rho_{0}}(u \sin \phi-v \cos \phi) \cos \phi \rightarrow$ (5)

$\frac{\partial \theta}{\partial t}+u \frac{\partial \theta}{\partial x}+v \frac{\partial \theta}{\partial y}=\alpha \nabla^{2} \theta+\frac{D_{F} c_{0} \theta_{0}}{C_{P}}+\left(\frac{\partial \mu_{s p}}{\partial c}\right)_{\theta_{0}, c_{0}} \cdot \nabla^{2} c \rightarrow$ (6)

$\frac{\partial c}{\partial t}+u \frac{\partial c}{\partial x}+v \frac{\partial c}{\partial y}=D \cdot \nabla^{2} c \rightarrow$
The appropriate initial and boundary conditions are:

For the whole space, at: $\mathrm{t}=0$.

$u=v=0, P=0,\left(\theta=\theta_{0}\right)$ and $\left(c=c_{0}\right)$ for $0 \leq x \leq L$ and $0 \leq y \leq L \rightarrow(8.1)$

At $\mathrm{t} \nmid 0$ :

$\left(\frac{\partial \theta}{\partial y}=\frac{\partial c}{\partial y}=0\right) \rightarrow(8.2)$

$u=v=0,\left(\theta=\theta_{h}\right)$ and $\left(c=c_{h}\right)$ for $x=0$ and $0 \leq y \leq L \rightarrow(8.3)$

$u=v=0,\left(\theta=\theta_{c}\right)$ and $\left(c=c_{c}\right)$ for $x=L$ and $0 \leq y \leq L \rightarrow(8.4)$

The set of the dimensionless governing equations using dimensionless variables defined below is, given by:

$\operatorname{div} U d=0 \rightarrow(9)$

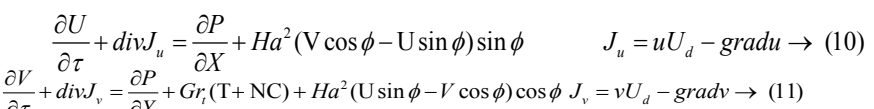

$\frac{\partial T}{\partial \tau}+d i v J_{T}=0 \quad J_{T}=T U_{d}-\frac{1}{\operatorname{Pr}} \operatorname{grad} T-\frac{D u}{\operatorname{Pr}} \cdot \operatorname{grad} C \rightarrow(12)$

$\frac{\partial C}{\partial \tau}+\operatorname{div} J_{C}=0 \quad J_{C}=C U_{d}-\frac{1}{S c} \operatorname{grad} C \rightarrow$

In this study, the height of the enclosure $L$ is taken as a reference length for the dimensionless variables. Thus, the dimensionless above equations are established using the following dimensionless variables:

$\tau=\frac{t}{L^{2}} \alpha ;(\mathrm{X}, \mathrm{Y})=\frac{(\mathrm{x}, \mathrm{y})}{L} ;(\mathrm{U}, \mathrm{V})=\frac{(\mathrm{u}, \mathrm{v})}{\alpha} L ; P=\frac{p L^{2}}{\rho_{0} \alpha^{2}} ; \mathrm{T}=\frac{\theta-\theta_{0}}{\theta_{h}-\theta_{c}} ; C=\frac{c-c_{0}}{c_{h}-c_{c}} ;$

$\theta_{0}=\frac{\theta_{h}-\theta_{c}}{2} ; c_{0}=\frac{c_{h}+c_{c}}{2} ; G r_{T}=\frac{g \beta_{T}\left(\theta_{h}-\theta_{c}\right) \mathrm{L}^{3}}{v^{2}} ; G r_{C}=\frac{g \beta_{C}\left(c_{h}-c_{c}\right) \mathrm{L}^{3}}{v^{2}} ; \mathrm{N}=\frac{G r_{c}}{G r_{T}} ;$

$H a^{2}=\frac{\sigma B_{0}^{2} L^{2}}{\mu} ; \operatorname{Pr}=\frac{v}{\alpha} ; L e=\frac{\alpha}{D} ; S c=L e \operatorname{Pr} ; D u=\frac{W D_{F} \beta_{T}}{\alpha \beta_{c}} \quad \rightarrow \quad(14)$

The initial and boundary conditions in dimensionless form that solve the stated Equations system (9-13) are:

For the whole domain, at $\tau=0$ :

$U=V=0, P=0(T=0.5-X)$ and $(C=0.5-X)$ for $0 \leq X \leq 1$ and $0 \leq Y$ $\leq 1 \rightarrow(15.1)$

As $\tau>0$

\begin{tabular}{lllll}
\multicolumn{1}{c}{ Conditions } & Velocity & Temperature & Concentration \\
\multicolumn{2}{ll}{ Bottom and top wall } & & & \\
Hot wall & $X=00 \leq Y \leq L$ & $U=V=0$ & $T=0.5$ & $C=0.5 \rightarrow(15.3)$ \\
Cold wall & $X=10 \leq Y \leq L$ & $U=V=0$ & $T=-0.5$ & $C=-0.5 \rightarrow(15.3)$
\end{tabular}

The average heat and mass transfer along the heated wall are given in dimensionless form by Nusselt and Sherwood numbers, respectively. They are defined as follow:

$$
\begin{aligned}
& N u=\int_{0}^{1}\left(\frac{\partial T}{\partial X}\right) d Y \rightarrow \\
& S h=\int_{0}^{1}\left(-\frac{\partial C}{\partial X}\right) d Y \rightarrow
\end{aligned}
$$

\section{Second law formulation}

Entropy generation of a system is the sum of Irreversibilities (degraded energy) that are produced due to different phenomena inside the system. The volumetric local entropy generation in double-diffusive convection through a square medium with the effect of magnetic field can be calculated by the following equation [10]. The first term of the 
right hand side of Equation (18) is irreversibility due to heat transfer, the second is due to mass transfer, the third to fluid friction and the fourth is relative to the magnetic field.

$$
\dot{S}_{g e n}=\frac{\lambda}{T_{0}^{2}}(\nabla \mathrm{C})^{2}+\frac{R D}{T_{0}}(\Delta \mathrm{C} \cdot \Delta \mathrm{T})+\frac{\vec{\tau}: \overline{\overline{\operatorname{grad}}} \vec{v}}{T}+\frac{\sigma_{e} B^{2}}{T_{0}}(\mathrm{u} \sin \alpha-\mathrm{v} \cos \alpha)^{2} \rightarrow(18)
$$

Total dimensionless entropy generation is obtained by a numerical integration of the dimensionless local entropy generation over the entire volume of the cavity, $\Omega$. It is given by:

$$
S=\int_{\Omega} \dot{S}_{\text {gen }} d \Omega \rightarrow(19)
$$

\section{Numerical method}

The dimensionless governing Equations (9) to (13) subjected to the boundary conditions (15) are integrated numerically using the control volume finite element method CVFEM [11,12]. The above equations are resolved by applying the SIMPLE algorithm of [11]. The SIMPLER algorithm and the SIMPLEC approximation of Van Doormal and Raithby are used in conjunction with an Alternating Direction Implicit (ADI) scheme for performing the time evolution. For more details, the used numerical code written in FORTRAN language and discussions about these functions, reader can see [13-16].

The power law scheme proposed by Patankar [11] is used for the convection terms formulation of the energy and mass transfer equations.

The resulting algebraic equations were solved by line-by-line using the Tri-Diagonal Matrix Algorithm iteration. We assumed linear and exponential variations when the dependent variable $\chi$ is calculated in the diffusive and the convective terms of the conservation equations, respectively. $\chi$ is the general dependent variable which can stands for $\mathrm{u}, \mathrm{v}, \mathrm{T}$, or C.

The iteration process is terminated under the following condition.

$$
\max \left|\frac{\chi^{t+\Delta t}-\chi^{t}}{\chi^{t+\Delta t}}\right| \leq 10^{-5} \rightarrow(20)
$$

The local volumetric entropy generation is calculated after calculating the temperature, concentration and stream function in each control volume in the mesh. Finally the entropy generation number for the cavity volume is calculated using Equation (9) via the numerical integration.

Results shown in the following section have been calculated by taking into account the initial and the boundary conditions given above. Table 1 depicts the used grids (i.e., sizes), the dimensionless times as well as the time steps that are found sufficient to reach the steady state situation and the imposed convergence criteria. Error percentage is given by:

$$
\operatorname{Er}(\%)=(|N u(x+10, x+10)-N u(x, x)| / N u(x, x)) \times 100
$$

It is found that $51 \times 51$ grid size is an appropriate size for the most calculations in the present problem, $x$ represents the Grid size. Comparison of the calculated average Nusselt number using the present numerical method for different thermal Grashof numbers with literature is presented by Davis and Nithyadevi [16,17] (see Table 2). For various Prandtl number values, Table 3 illustrates a comparison of average Nusselt number results with those published by Pesso et al. [18] at fixed thermal Grashof number, $G r_{T}=10^{5}$. Isotherms and streamlines are plotted in Figure 2 for different thermal Grashof number and compared to results obtained by Abbassi et al. [13] For thermosolutal natural convection in a square cavity, the present simulation is validated against numerical results of Sezai and Teamah $[18,19]$ for different Lewis numbers and Buoyancy ratios. Figures 3 and 4 illustrate a validation by; avearage Nusselt and Sherwood numbers versus Lewis number and versus Buoyancy ratio variations and by isotherms and isoconcentrations contours at fixed Rayleigh number, $R a=10^{5}$, Prandtl number, $P r=10$ and Buoyancy ratio, $N=-0.2$ [20-23].

Relatively good agreement is obtained. We are, therefore, confident that our results are accurate.

\section{Results and Discussion}

The present work focused the study of the fluid flow structure and entropy generation in double-diffusive natural convection through a square cavity submitted to an oriented magnetic field with Dufour effect

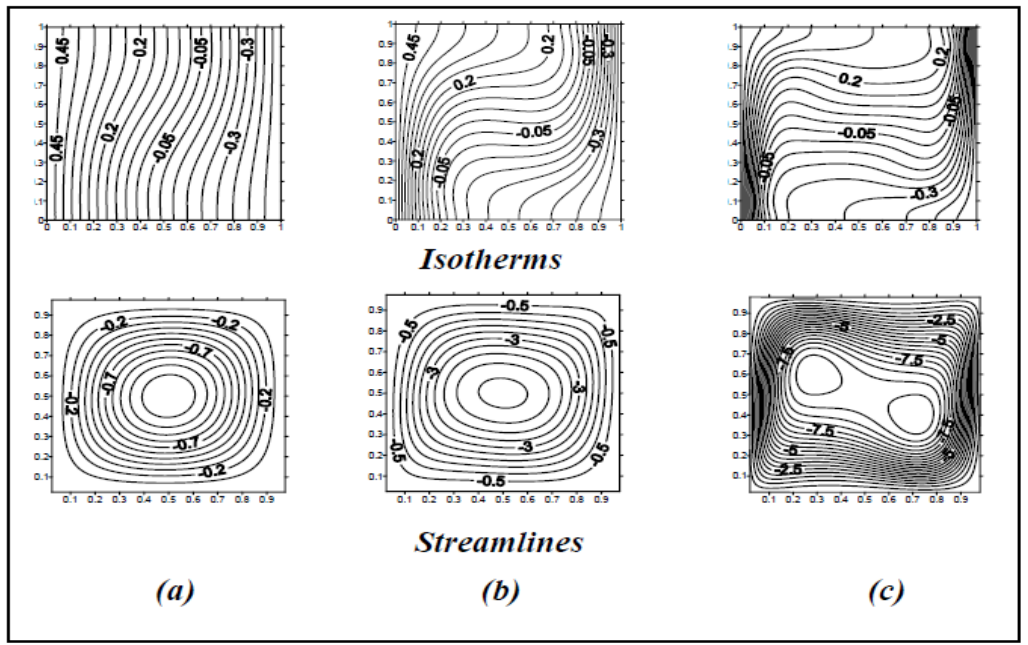

Fig.2

a) $\mathrm{Ra}=10^{3}$. b) $\mathrm{Ra}=10^{4}$. c) $\mathrm{Ra}=10^{5}$.

Figure 2: Isotherms and Streamlines $(\operatorname{Pr}=0.71, \mathrm{Le}=2)$. 


\begin{tabular}{|c|c|c|c|}
\hline Grid size & Nu & Er (\%) & Ra=Gr $\times$ Pr \\
\hline $21 \times 21$ & 1.1024 & -- & \multirow{2}{*}{$10^{3}$} \\
\hline $31 \times 31$ & 1.0992 & 0.29 & \\
\hline $41 \times 41$ & 1.0979 & 0.408 & \multirow{2}{*}{$10^{4}$} \\
\hline $31 \times 31$ & 2.3201 & -- & \\
\hline $41 \times 41$ & 2.2956 & 1.055 & $10^{5}$ \\
\hline $51 \times 51$ & 2.2832 & 1.59 & \\
\hline $41 \times 41$ & 4.7097 & -- & \\
\hline $51 \times 51$ & 4.6641 & 0.968 & \\
\hline $61 \times 61$ & 4.6593 & 1.07 & \\
\hline
\end{tabular}

Table 1: Grid size independence study $(\mathrm{Pr}=0.71, \mathrm{~N}=\mathrm{Ha}=0)$.

\begin{tabular}{|c|c|c|c|}
\hline Ra & $10^{3}$ & $10^{4}$ & $10^{5}$ \\
\hline Present study & 1.099 & 2.295 & 4.664 \\
\hline Davis & 1.118 & 2.243 & 4.519 \\
\hline Nithyadevi et al. & 1.123 & 2.304 & 4.899 \\
\hline
\end{tabular}

Table 2: Average Nusselt number for different values of Rayleigh number $(\mathrm{Pr}=0.71$, $\mathrm{N}=\mathrm{Ha}=0$ ).

\begin{tabular}{|c|c|c|c|c|}
\hline Pr & 0.071 & 0.71 & 1 & 7.1 \\
\hline Present study & 3.948 & 4.664 & 4.745 & 4.863 \\
\hline Pesso et al. & 3.813 & 4.521 & 4.685 & 4.732 \\
\hline Relative error (Er(\%)) & 3.54 & 3.16 & 1.28 & 2.77 \\
\hline
\end{tabular}

Table 3: Average Nusselt number for different values of Prandtl numbers $\left(\mathrm{Gr}_{\mathrm{T}}=10^{5}\right.$, $\mathrm{N}=\mathrm{Ha}=0$ ).

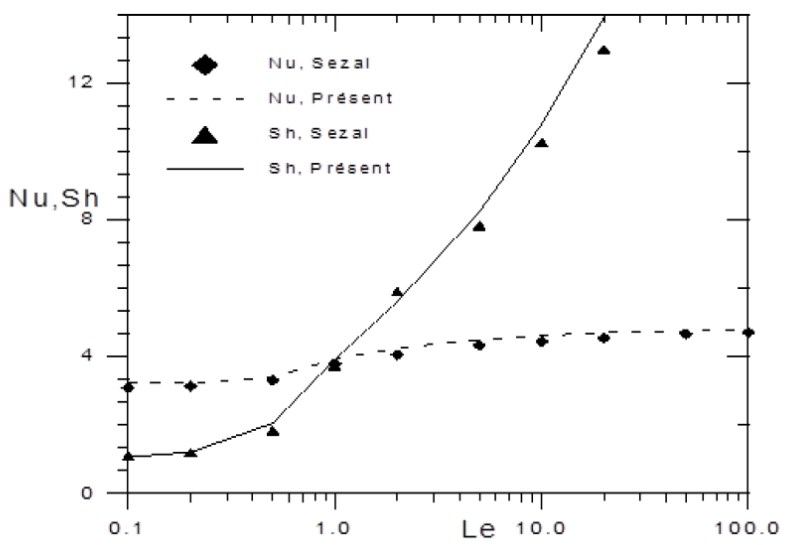

Figure 3: Average Nusselt and Sherwood numbers versus Lewis number $\left(\mathrm{Ra}=10^{5}, \mathrm{~N}=-0.2, \mathrm{Pr}=10\right)$.

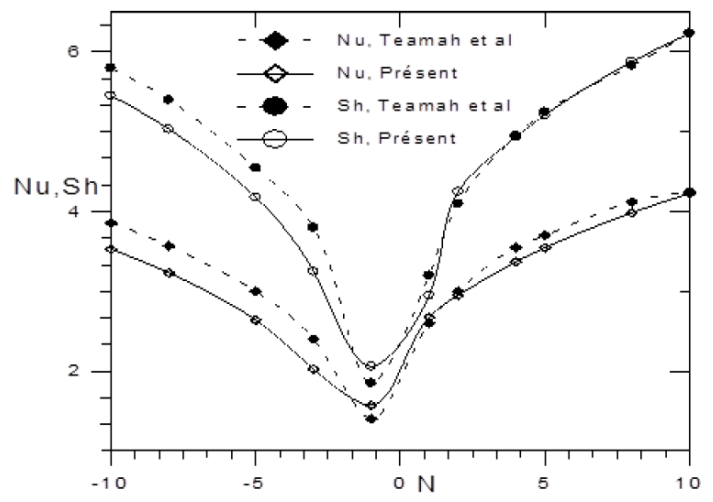

Figure 4: Average Nusselt and Sherwood numbers versus Buoyancy ratio $\left(\mathrm{Ra}=10^{4}, \mathrm{~N}=-0.2, \mathrm{Pr}=0.71, \mathrm{Le}=2\right)$. and saturated by a binary perfect gas mixture of air and a pollutant species characterized by $\operatorname{Pr}=0.71$ and $L e=1.2$.

Numerical computations of double-diffusive natural convection in a two-dimensional vertical square cavity in the presence of Magnetic and Dufour effects have been carried out for different values of $\mathrm{N}, \mathrm{Ha}$, $\mathrm{Du}$, and for fixed values of $\operatorname{Pr}=0.71$, which corresponds to air at $20^{\circ} \mathrm{C}$ and 1 atmospheric pressure, $\varnothing=0^{\circ}, \mathrm{Le}=1.2$ and $\mathrm{RaT}=105$. With the abovementioned flow parameters, the results are displayed in Figures 5-10, for the velocity, temperature and concentration profiles and for entropy generation rate behaviour. The rate of heat and mass transfer in the enclosure is measured in terms of the average Nusselt number and average Sherwood number.

The Dufour effect consists on concentration grdients that drive heat currents or change the temperature field on convection in binary heated fluid layers. The Dufour effect changes the temperarure field equation "diagonally" via the term $\left(-\Delta^{2} \mathrm{~T}\right)$ which enhances heat diffusion and "off-diagonally" via the term $\left(-\Delta^{2} \mathrm{C}\right)$ which reflects concentrationinduced temperature variations.

The effects of Dufour parameter on the velocity field for $N=-1$, $H a=0$ and $R a_{T}=10^{5}$ are shown in Figure 5. It exhibits a comparison of center line velocity profiles along $\mathrm{x}$ and $\mathrm{y}$ directions at the horizontal and vertical center lines in the cavity for different values of $D u$. the velocity is substantially zero in the absence of the Dufour effect $(D u=0)$ indicating a state of stable equilibrium. Increasing $D u$ leads to an increase in the fluid velocity field by means of an increase in the temperature boundary layer thickness due to augmentation of the fluid temperature which is proportional to Dufour effect, as a result the birth of a convective flow following the disappearance of the diffusive flow. The increase in velocity with Dufour parameter indicates that the buoyant convection can be enhanced by an increase in $D u$. these results may be explained by considering the temperature trend as depicted in Figure 6a. The influence of Dufour effect on the concentration field is illustrated in Figure 6b.

Figure 7 displays the variations of entropy generation rate (S) on the buoyancy ratio $(\mathrm{N})$ at two Hartmann number values $(\mathrm{Ha}=0$ and $\mathrm{Ha}=25)$ for different Dufour parameter $(\mathrm{Du})$. It can be noticed that the amplitude of the entropy generation increases (respectively decreases) with the Dufour parameter in the case of cooperating buoyancy forces (respectively opposing case) whereas it decreases with Hartmann number increasing. The entropy generation takes a minimum value obtained at $\mathrm{N}=-1$. Dufour effect causes a significant increase in irreversibility due to friction. Therefore the movement of the fluid within the cavity is high, resulting from an increase in the fluid velocity field caused by the buoyancy forces. Increasing Hartmann number leads to reduce the flow circulation strength in the cavity and this leads to decrease the rate of heat and mass transfer as well as the rate of entropy generation. Enhancing the magnetic effect leads to a decrease in the velocity field as can be seen in Figure 8, the centreline velocities are signicantly reduced due to a development of MHD Hartmann boundary layer. The magnetic field presents a damping effect on the velocity by creating a drag force that tends to suppress the fluid flow.

The effects of the Dufour parameter $(\mathrm{Du})$ on the average Nusselt and Sherwood numbers and entropy generation rate at fixed buoyancy ratio $(\mathrm{N}=-1)$ are presented in Figure 9 which shows that for absence of magnetic field $(\mathrm{Ha}=0)$, when the Dufour parameter increases, they increase. Additionally, for moderate magnetic effect $(\mathrm{Ha}=25)$, the average Nusselt and Sherwood numbers slightly decrease until $\mathrm{Du}=0.2$, then they increase as Dufour parameter increases. For high values of Hartmann number $(\mathrm{Ha} \geq 100)$, the average Nusselt and Sherwood 
Citation: Bouabid M, Hidouri N, Magherbi M, Brahim AB (2017) Heat and Mass Transfer for Hartmann and Dufour's Effects on Irreversibilities at Double-Diffusive Natural Convection in a Square Cavity. J Adv Chem Eng 7: 180. doi: 10.4172/2090-4568.1000180

Page 6 of 11

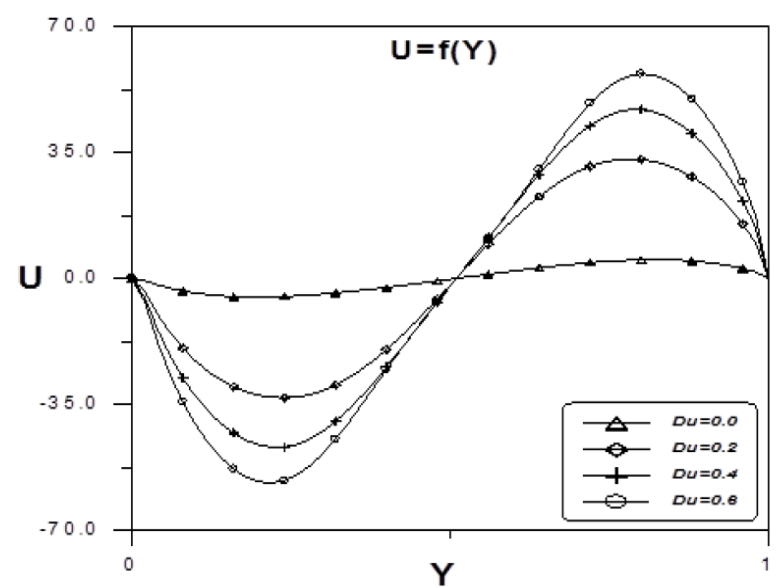

Figure 5a: Velocity profiles in $\mathrm{x}$ direction at $\mathrm{x}=0.5$ versus Dufour parameter $\left(\mathrm{Ra}=10^{5}, \mathrm{~N}=-1, \mathrm{Ha}=0\right)$.

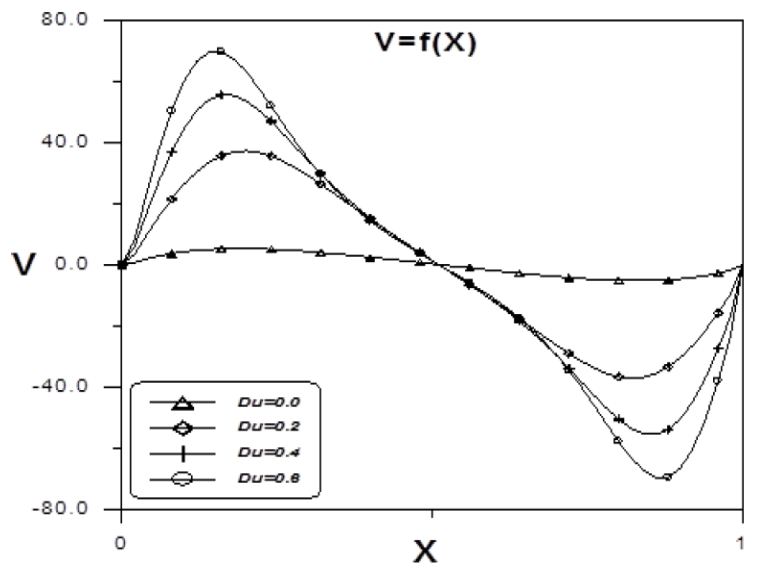

Figure $5 \mathrm{~b}$ : Velocity profiles in y direction at $\mathrm{y}=0.5$ versus Dufour parameter $\left(\mathrm{Ra}=10^{5}, \mathrm{~N}=-1, \mathrm{Ha}=0\right)$.

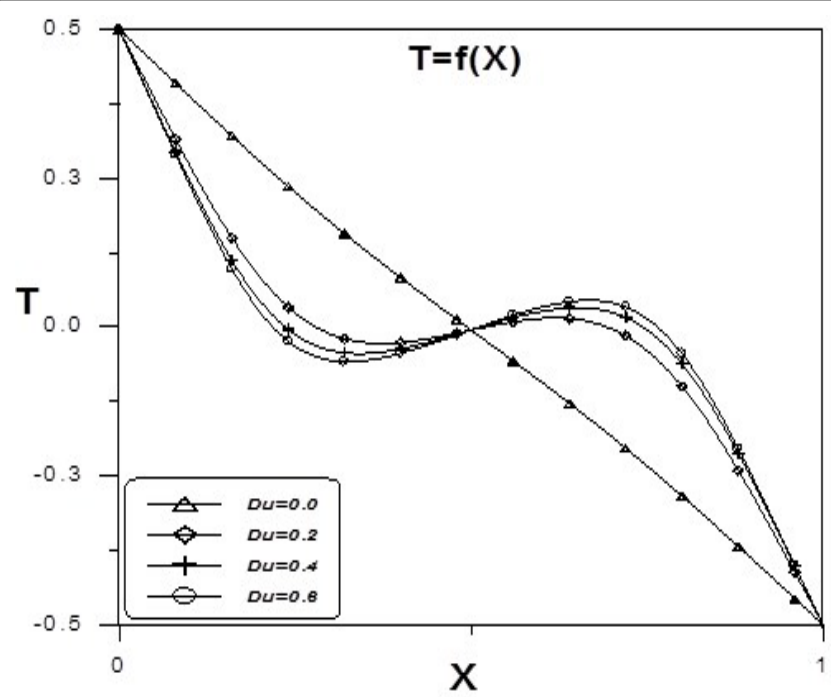

Figure 6a: Temperature profiles versus Dufour parameter $\left(\mathrm{Ra}=10^{5}, \mathrm{~N}=1\right.$, $\mathrm{Ha}=0$ ).

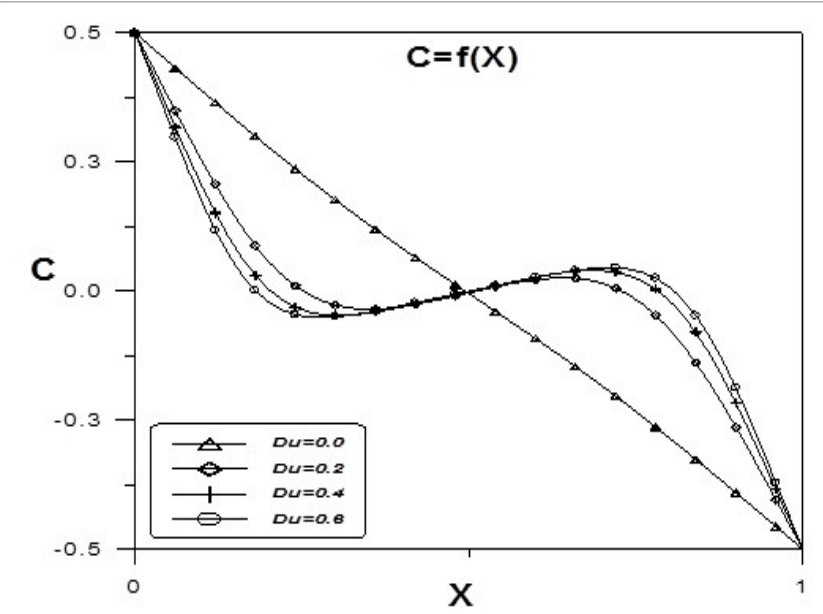

Figure 6b: Concentration profiles versus Dufour parameter $\left(R a=10^{5}, N=-1\right.$, $\mathrm{Ha}=0$ ).

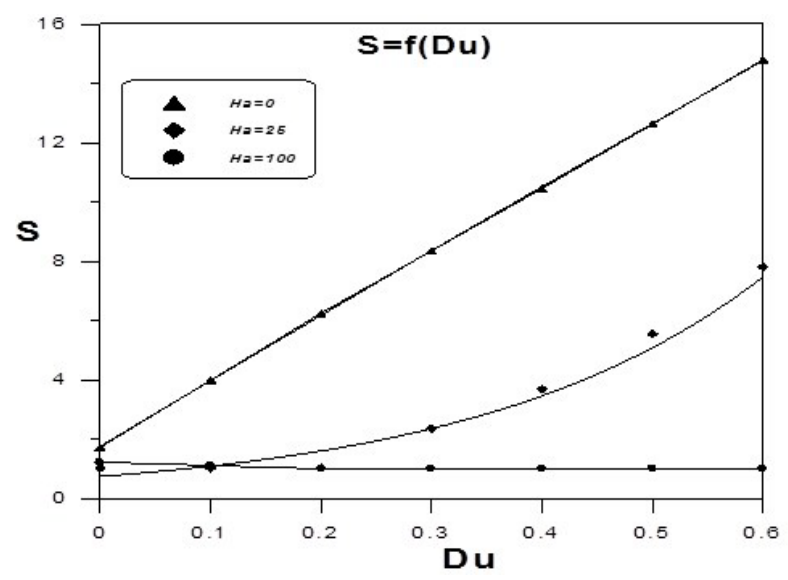

Figure 7: Entropy generation rate versus Dufour parameter for different Hartmann number $\left(\mathrm{Ra}=10^{5}, \mathrm{~N}=-1\right)$.

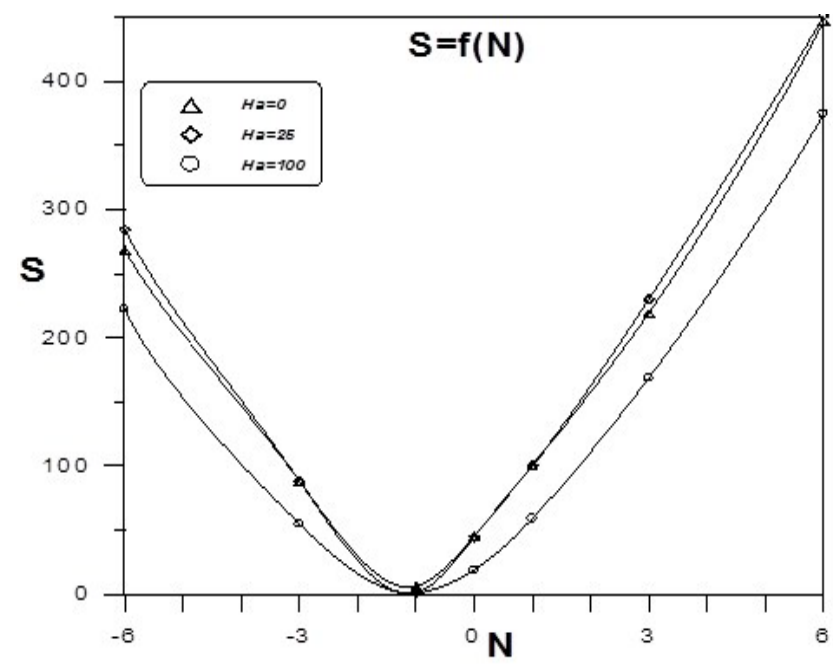

Figure 8a: Average Nusselt number versus Buoyancy ratio for different Hartmann number $\left(\mathrm{Ra}=10^{5}, \mathrm{Du}=0.2\right)$. 
numbers decrease until $\mathrm{Du}=0.2$, then they are no influenced by Dufour effect and remain constant approching unity. The entropy generation ratio shows a monotonic behaviour with Dufour increase for low magnetic effect, exponetial trend for moderate magnetic field and no significant effect at high values of Hartmann number.

Figure 10 shows the variations of average Nusselt $(\mathrm{Nu})$ and Sherwood (Sh) numbers and entropy generation rate $(\mathrm{S})$ with buoyancy ratio $(\mathrm{N})$ for different Hartmann number $(\mathrm{Ha})$ at fixed value of Dufour parameter $(\mathrm{Du}=0.2)$. Increasing the magnetic field leads to increase the Lorentz forces which tends to suppress the fluid flow leading to a decrease of $\mathrm{Nu}$ and $\mathrm{Sh}$ rapidly towards unity, therefore the entropy generation decreases but it increases with the absolute value of buoyancy ratio, N. For all the values of $\mathrm{Ha}$, minimum values of $\mathrm{Nu}$, $\mathrm{Sh}$ and $\mathrm{S}$ are obtained at $\mathrm{N}=-1$ which means that the buoyancy forces generated due to temperature and concentration gradients are acting equally in opposite directions. This case corresponds to stagnant fluid where the heat and mass transfers are in pure diffusion processes.

Figure 11 show the effect of Hartmann number at fixed Dufour parameter, $R a_{T}=10^{5}$ and $N=-1$ on isotherms, streamlines and isentropic lines. As magnetic effect increases, it is observed that the vortex strength increases, the streamline behaviour is very pronounced at high Hartmann numbers $(H a=100)$, it can be seen that the streamline is elongated and two secondary vortices appear inside the cavity. These influences on the vortex strength are proportional to the Dufour parameter increasing. The flow is driven by the buoyancy force due to the effect of both temperature and concentration variations and draged by Lorentz force which has a retarding effect to the fluid flow.

For low Hartmann number situations, most of the buoyancy driver is due to the effect of temperature variations. The effects of Hartmann number are clearly seen on the isotherms at moderate and high Dufour parameter, it could be seen that for low Hartmann number, the isotherms are nearly parallel near the bottom left wall and near the upper right corner as a result of the fluid rising due to the influence of the buoyancy force. Further increase in Ha eventually leads to a linear temperature variation between the hot and cold regions, almost like heat conduction in a solid. The isotherm lines become parallel corresponding to pure conduction regime. An increase in Dufour parameter enhance entropy generation in the enlosure where its maximum is concentrated near the hot and cold walls but with increasing magnetic effect, isentropic lines prolfie tends to cover the whole domain. This is due to the anisotropic nature of $M H D$ flow.

\section{Conclusions}

The influence of magnetic and Dufour effects on entropy generation inside a square cavity filled with a binary perfect gas mixture at doublediffusive convection is accounted. Various Hartmann numbers, Buoyancy ratios and Dufour parameters have been considered for the flow, temperature and concentration fields as well as the entropy generation rate. The results of the numerical analysis lead to the following conclusions:

- Increasing Du leads to an increase in the fluid velocity field by means of an increase in the temperature boundary layer thickness,

- The amplitude of the entropy generation increases (respectively decreases) with the Dufour parameter in the case of cooperating buoyancy forces (respectively opposing case),

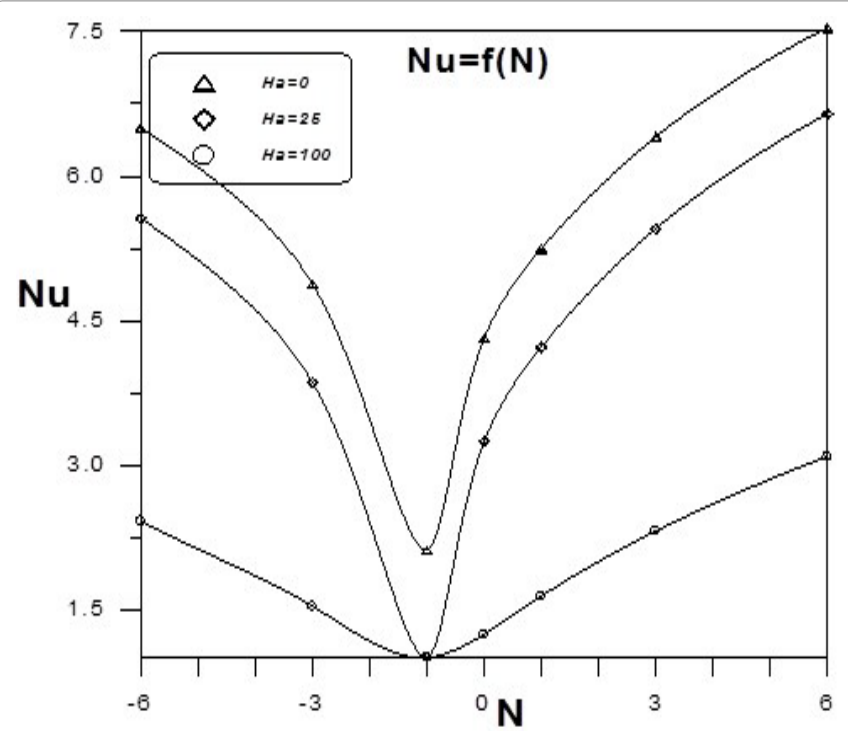

Figure 8b: Average Sherwood number versus Buoyancy ratio for different Hartmann number $\left(\mathrm{Ra}=10^{5}, \mathrm{Du}=0.2\right)$.

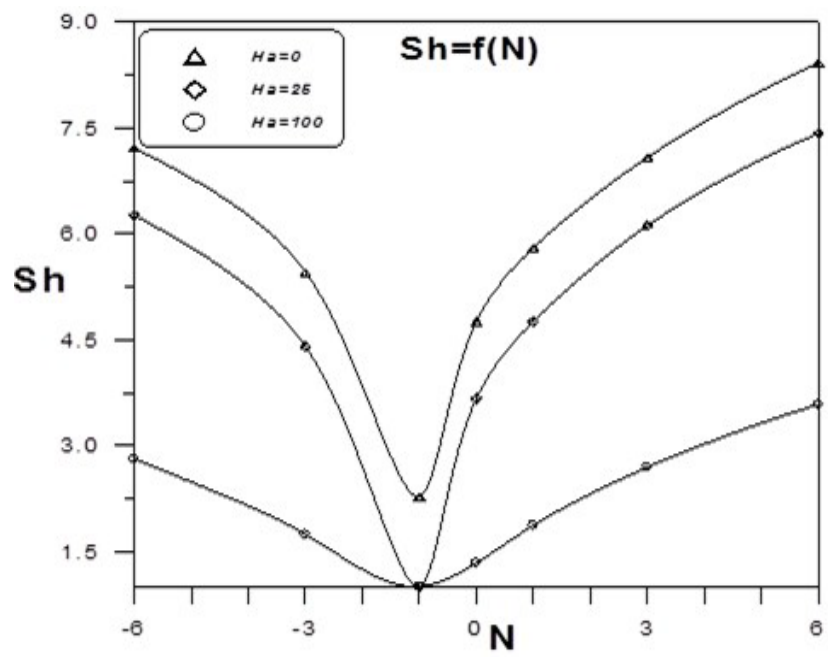

Figure 8c: Average Sherwood number versus Buoyancy ratio for different Hartmann number $\left(\mathrm{Ra}=10^{5}, \mathrm{Du}=0.2\right)$.

- The magnetic field presents a damping effect on the velocity by creating a drag force that tends to suppress the fluid flow,

- The entropy generation ratio shows a monotonic behaviour with Dufour increase for low magnetic effect, exponetial trend for moderate magnetic field and no significant effect at high values of Hartmann number,

- For all the values of $\mathrm{Ha}$, minimum values of $\mathrm{Nu}, \mathrm{Sh}$ and $\mathrm{S}$ are obtained at $\mathrm{N}=-1$,

- The structure of the fluid streamlines, isotherms and isoentropic lines within the enclosure is found to significantly depend upon the $\mathrm{Ha}$ and $\mathrm{Du}$.

\section{Acknowledgements}

The authors are grateful to a referee and an editor for great suggestions and help in polishing the manuscript. 

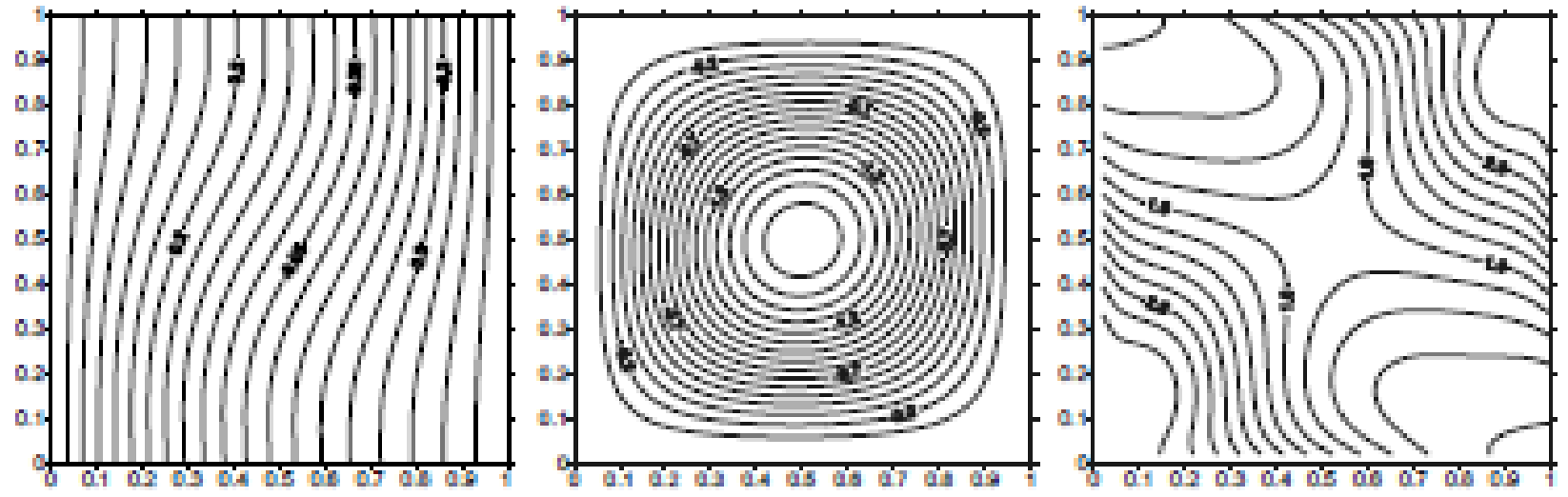

$H a=0$
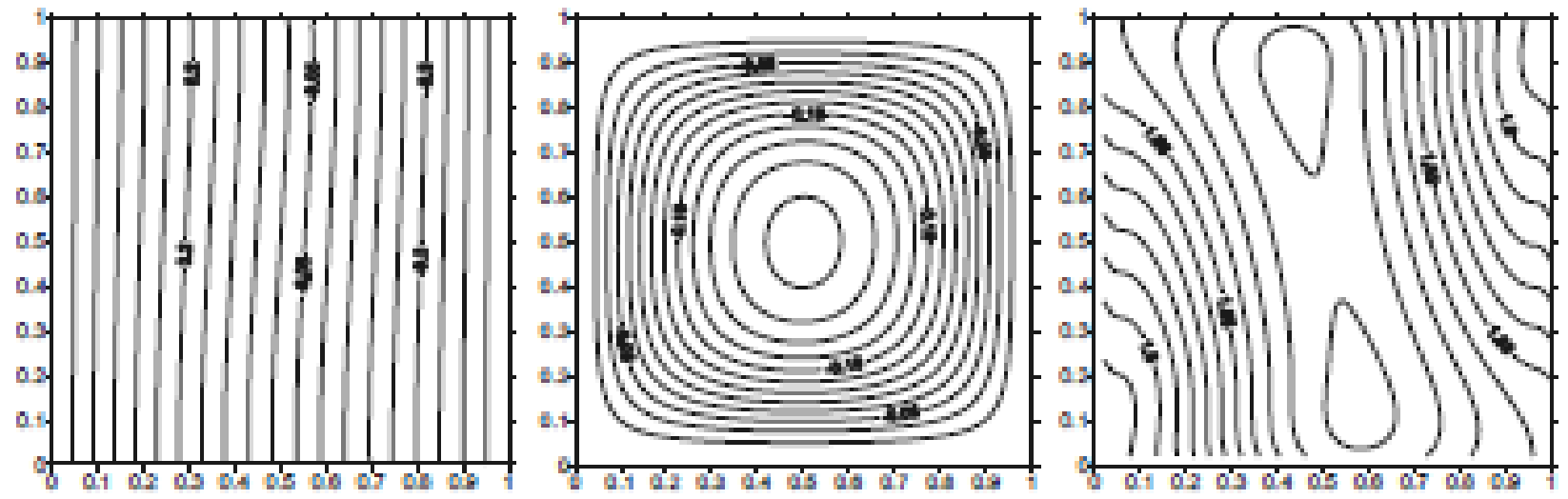

$H a=25$
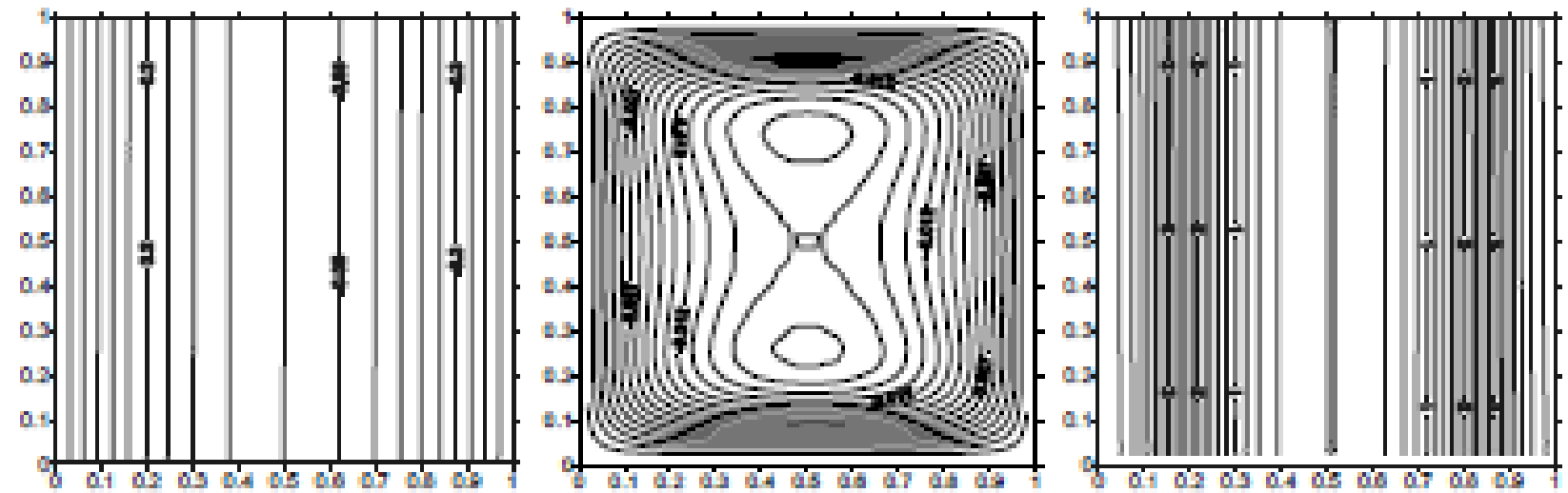

$H a=100$

Inotherms

Streambnes

Ischuropie lones

Figure 9: Isotherms, streamlines and isentropic lines for different Hartmann number $\left(\operatorname{Ra}_{\mathrm{T}}=10^{5}, \mathrm{~N}=-1, \operatorname{Pr}=0.71, \mathrm{Le}=1.2, \mathrm{Du}=0\right)$. 

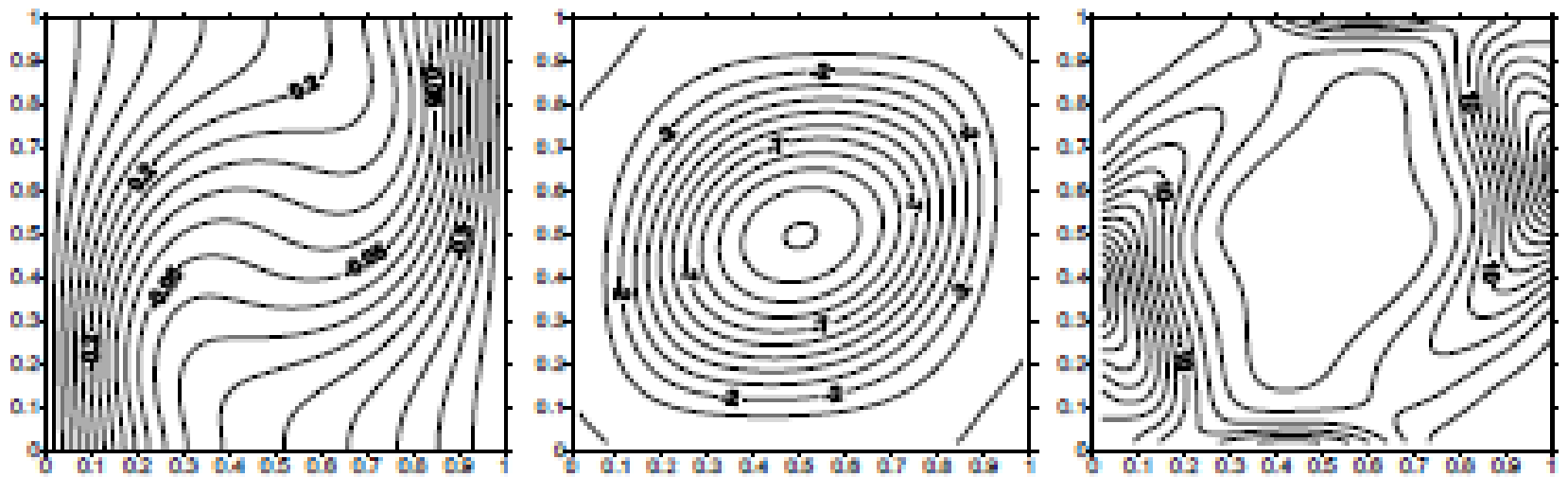

$H a=0$
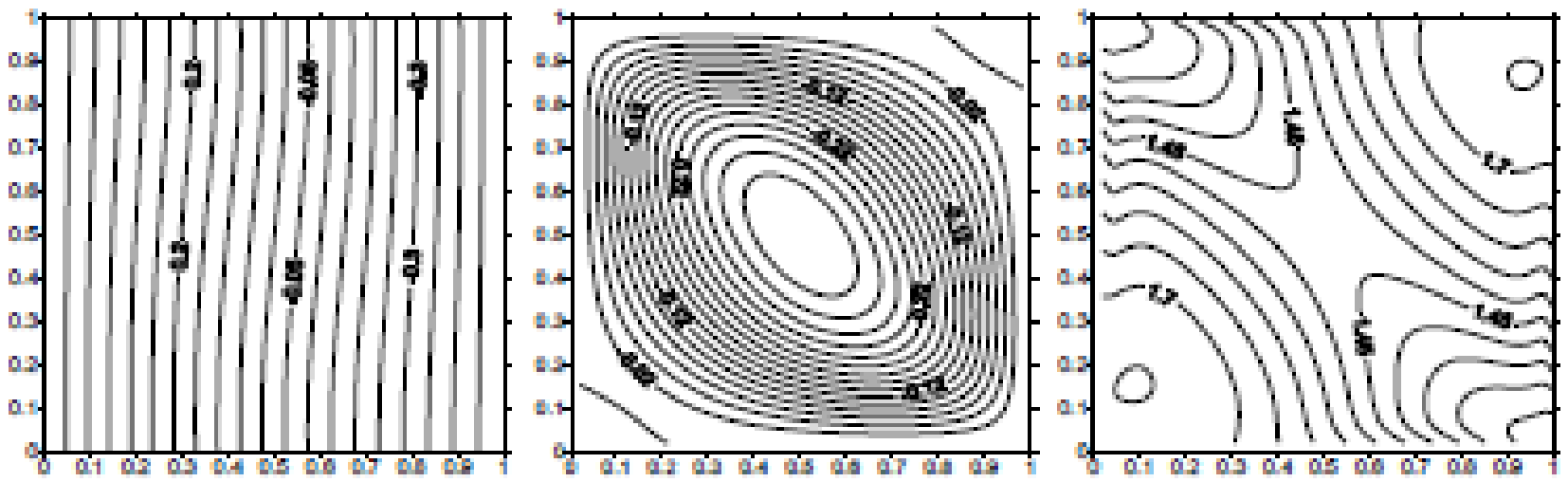

$\mathrm{Ha}=25$
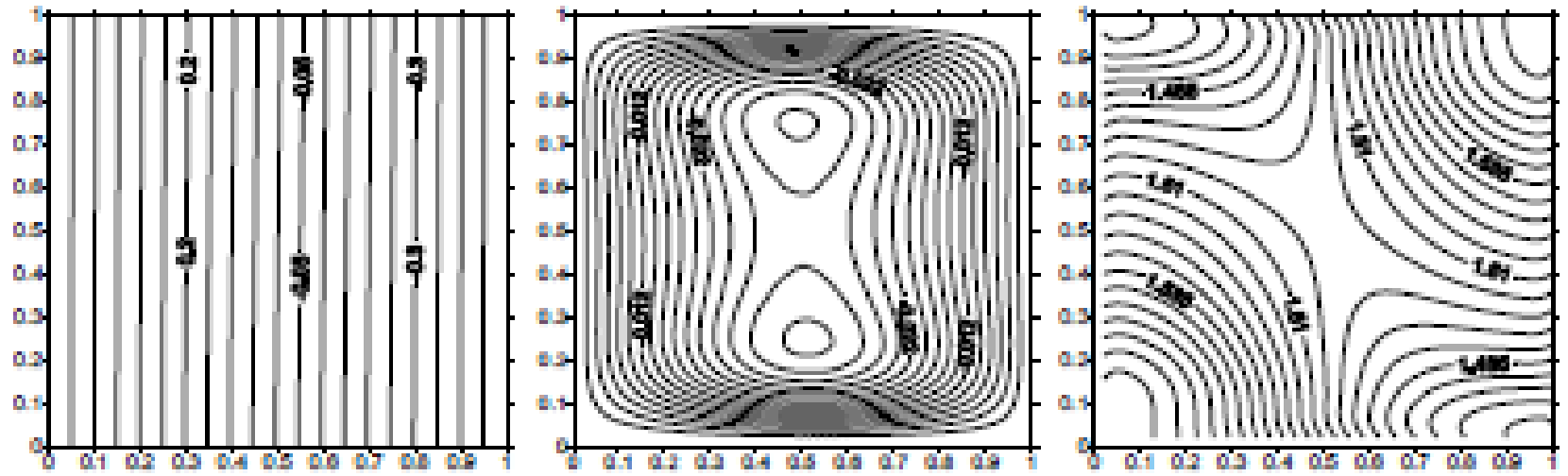

$H a=100$

Totherms

Sureambnes

Isentropie thes

Figure 10: Isotherms, streamlines and isentropic lines for different Hartmann number $\left(\operatorname{Ra}_{\mathrm{T}}=10^{5}, \mathrm{~N}=-1, \operatorname{Pr}=0.71, \mathrm{Le}=1.2, \mathrm{Du}=0.2\right)$. 

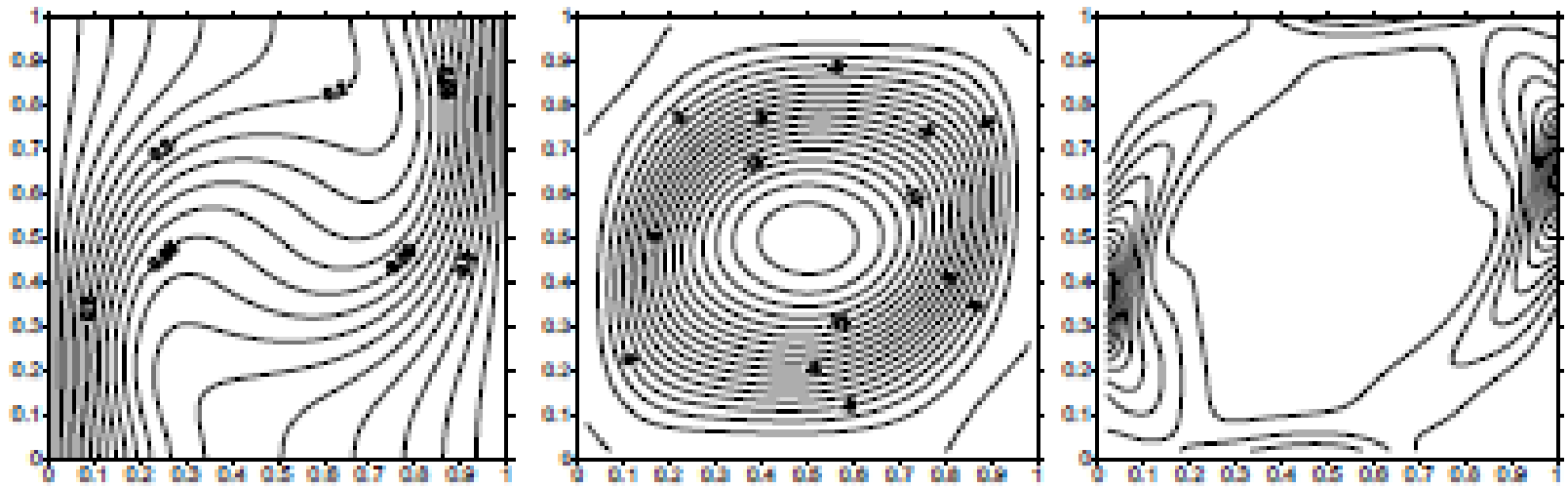

$H a=0$
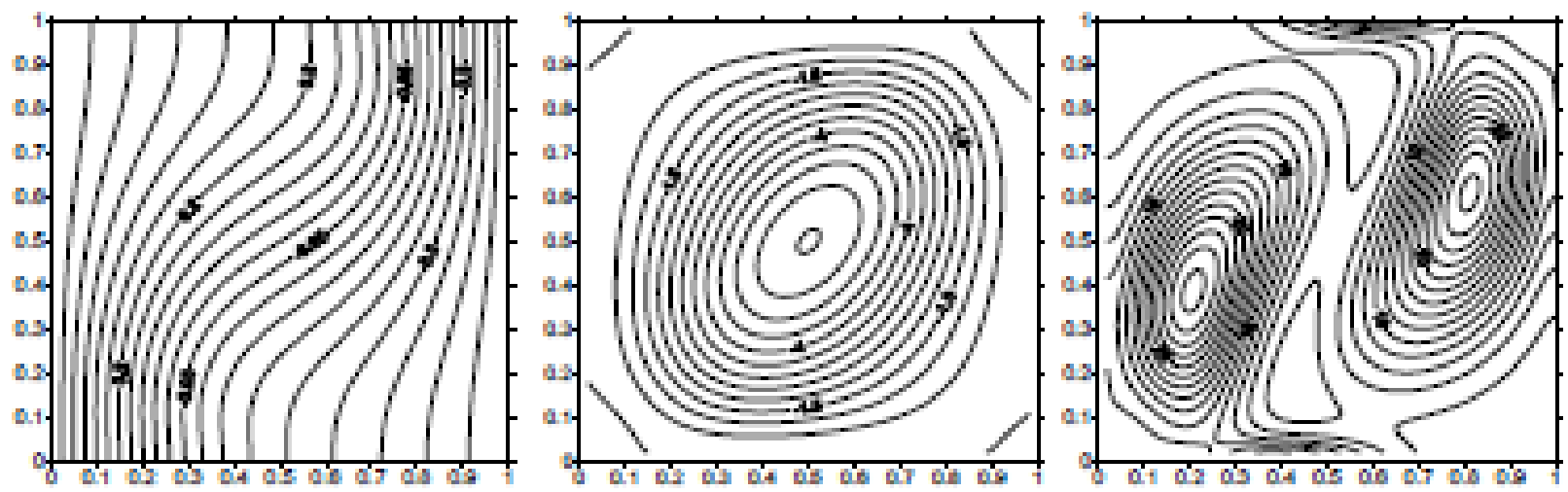

\section{$H a=25$}
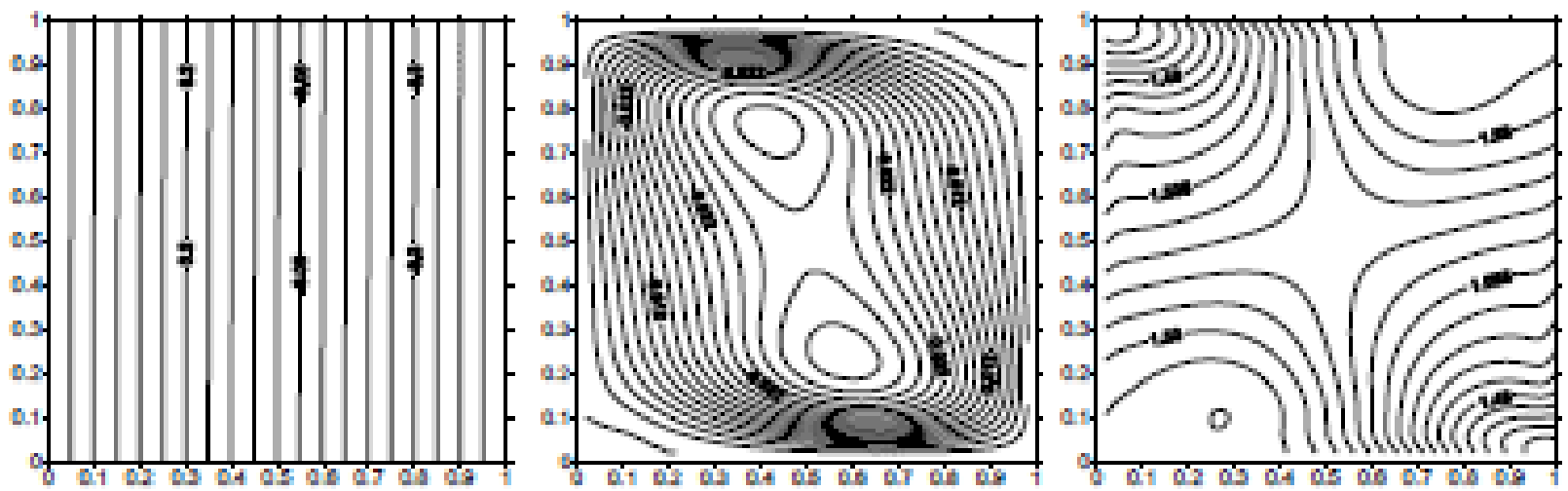

$H a=100$

Trohtrins

Streamines

Inenapopic lines

Figure 11: Isotherms, streamlines and isentropic lines for different Hartmann number $\left(\operatorname{Ra}_{\mathrm{T}}=10^{5}, \mathrm{~N}=-1, \mathrm{Pr}=0.71, \mathrm{Le}=1.2, \mathrm{Du}=0.6\right)$. 
Citation: Bouabid M, Hidouri N, Magherbi M, Brahim AB (2017) Heat and Mass Transfer for Hartmann and Dufour's Effects on Irreversibilities at Double-Diffusive Natural Convection in a Square Cavity. J Adv Chem Eng 7: 180. doi: 10.4172/2090-4568.1000180

Page 11 of 11

\section{References}

1. Trevisan OV, Bejan A (1985) Combined heat and mass transfer by natura convection in porous medium. IJHMT 28: 909-918.

2. Ali M, Alam MS (2013) Soret and Dufour Effects on Steady free Convection in MHD Micropolar Fluid Flow, Mass and Heat Transfer with Hall Current. IJART 2: $130-138$.

3. Subhakar MJ, Gangadhar K, Reddy NB (2012) Soret and dufour effects on MHD convective flow of heat and mass transfer over a moving non-isothermal vertical plate with heat generation/absorption. AASR 3: 3165-3184.

4. Sharma BK, Yadav K, Mishra NK, Chaudhary RC (2012) Soret and Dufour Effects on Unsteady MHD Mixed Convection Flow past a Radiative Vertical Porous Plate Embedded in a Porous Medium with Chemical Reaction. Applied Mathematics 3: 717-723.

5. Bouabid M, Hidouri N, Magherbi M, Brahim AB (2011) Analysis of the Magnetic Field Effect on Entropy Generation at Thermosolutal Convection in a Square Cavity. Entropy 13: 1034-1054.

6. Hidouri N, Magherbi M, Abbassi H, Brahim AB (2007) Entropy generation in double diffusive in presence of Soret effect. PCFD 5: 237-246.

7. Magherbi M, Abbassi $H$, Hidouri N, Brahim AB (2006) Second law analysis in convective heat and mass transfer. Entropy 8: 1-17.

8. Malashetty MS, Gaikwad SN (2002) Effect of cross diffusion on double diffusive convection in the presence of horizontal gradients. IJES 40: 773-787.

9. Ahammad MU, Mollah MS (2011) Numerical Study of MHD Free Convection Flow and Mass Transfer Over a Stretching Sheet Considering Dufour \& Soret Effects in the Presence of Magnetic Field. IJET IJENS 5: 3-8.

10. Woods LC (1975) The Thermodynamics of Fluid Systems. Oxford University Press, Oxford, UK.

11. Patankar SV (1980) Numerical heat transfer and fluid flow. In: Computational Methods in Mechanics and Thermal Sciences. Hemisphere/MacGraw Hill. New
York, USA.

12. Saabas HJ, Baliga BR (1994) Co-located equal-order control volume finite element method for multidimensional incompressible fluid flow. Part I: FNHT Pt. B-Fund 26: 381-407.

13. Abbassi $H$, Turki S, Ben NS (2001) Numerical investigation of forced convection in a plane channel with a built-in triangular prison. IJTS 40: 649-658.

14. Nithyadevi N, Yang RJ (2009) Double diffusive natural convection in a partially heated enclosure with Soret and Dufour effects. IJHF 30: 902-910.

15. Davis VGD (1983) Natural convection of air in a square cavity, a bench mark numerical solution. IJNMF 3: 249-264.

16. Nithyadevi N, Yang RJ (2009) Magneto convection in an enclosure of water near its density maximum with Soret and Dufour effects. IJHMT 52: 1667-1676.

17. Pesso T, Piva S (2009) Laminar natural convection in a square cavity: Low Prandtl numbers and large density differences. IJHMT 52: 1036-1043.

18. Sezai I, Mohamad AA (2000) Double diffusive convection in a cubic enclosure with opposing temperature and concentration gradients. Phys Fluids 12: $2210-$ 2223.

19. Teamah MA, Dawood MMK, El-Maghlany WM (2011) Double Diffusive Natural Convection in a Square Cavity with Segmental Heat Sources. European Journal of Scientific Research 54: 287-301.

20. Mohan H, Kumar P (2014) Dufour - Driven Generalized MHD Double - Diffusive Convection. App Sci Report 8: 117-124.

21. Van DJP, Raithby GD (1984) Enhancements of the SIMPLE method for predicting incompressible fluid flow. Numer Heat Transfer 7: 147-163.

22. Abbassi H, Turki S, Ben NS (2001) Mixed convection in a plane channel with a built-in triangular prison. HTPA 39: 307-320.

23. Hollinger S, Lucke M (1995) Influence of the Dufour effect on convection in binary gas mixtures. Physical Review E 52: 642-657. 